\title{
ON THE INTEGRABILITY \\ OF SINGULAR DIFFERENTIAL FORMS IN TWO COMPLEX VARIABLES
}

\author{
A. MEZIANI
}

\begin{abstract}
In this work we study the integrability of a germ at $0 \in \mathbf{C}^{2}$ of a singular differential form for which the closure of the integral curves are analytic varieties that pass through 0 . The focii of this paper are the existence of pure meromorphic integrals, the linearization and the nonexistence of a topological criterion for transcendental integrability.
\end{abstract}

\section{INTRODUCTION}

Suppose that $\mathbf{w}$ is a germ at $0 \in \mathbf{C}^{2}$ of a holomorphic differential form that vanishes only at 0 . Then $\mathbf{w}$ generates a foliation $\mathbf{F}_{\mathbf{w}}$ in $U-\{0\}$, where $U$ is a small neighborhood of 0 in $\mathbf{C}^{2}$ : the leaves of $\mathbf{F}_{\mathbf{w}}$ are the integral curves of the differential equation $\mathbf{w}=0$. The properties of such foliations have been studied by several authors: C. Camacho, D. Cerveau, J. F. Mattei, R. Moussu, P. Sad and others. Among these foliations, the simplest ones are those defined by the connected components of the level sets of a function $f$. In this case $f$ is said to be an integral of $\mathbf{w}$ and satisfies $\mathbf{w} \wedge d f=0$.

In [6], J. F. Mattei and R. Moussu characterize holomorphic integrability. They prove that $\mathbf{w}$ has a holomorphic integral if and only if the closure (in a neighborhood of 0 ) of the integral curves are analytic varieties and only a finite number of them pass through 0 .

There is no such criterion for the existence of pure meromorphic integrals. By pure meromorphic function, we mean the quotient $\alpha / \beta$ of two nonunit coprime holomorphic functions $\alpha, \beta$. D. Cerveau and J. F. Mattei construct in [2] a homeomorphism of a neighborhood of $0 \in \mathbf{C}^{2}$ that maps the integral curves of the differential form

$$
\mathbf{u}=\left(y^{3}+y^{2}-x y\right) d x-\left(2 x y^{2}+x y-x^{2}\right) d y
$$

with no meromorphic integral (see [13]), into that of

$$
\mathbf{v}=\left(2 y^{2}+x^{3}\right) d x-2 x y d y
$$

which admits $\left(2 y^{2}+x^{3}\right) / x^{2}$ as a meromorphic integral. Hence they conclude the nonexistence of a topological criterion for the meromorphic integrability.

Received by the editors November 29, 1988.

1980 Mathematics Subject Classification (1985 Revision). Primary 58F18; Secondary 57R30. 
In this paper, we investigate the integrability of a class of differential forms. The closure of the integral curves of these differential forms are analytic varieties that pass through 0 . We use the term well-behaved for such foliations or differential forms. Note that the foliations defined by the level sets of pure meromorphic functions are examples of well-behaved foliations.

$\S 1$ is devoted to the necessary background. After recalling the notions of singular foliations and of the blow-up of a point on a two-dimensional complex manifold, we give a brief description of the desingularization of a holomorphic differential form. Then we define various integrals (holomorphic, meromorphic, transcendental and multiform) and recall some old results. Most of the material in this section can be found in [1] and [2].

In $\S 2$ we prove, as a consequence of the reduction theorem [2] and a linearization theorem for reduced forms [6], a reduction theorem for well-behaved differential forms. We show that if $\mathbf{w}$ is well behaved, then the foliation defined by $E^{*} \mathbf{w}(E: \mathbf{M} \rightarrow U$ is the reduction map) is conjugate near each one of its singularities to a foliation defined by

$$
p v d u+q u d v,
$$

where $p, q$ are coprime positive integers. Then we apply this result to reformulate the problem of finding an integrating factor $\rho$ of $\mathbf{w}$ (that is, $\rho$ is a holomorphic or a meromorphic function such that $\rho \mathbf{w}$ is a closed form) into solving a kind of Cousin problem in the unit disc.

$\S 3$ deals with the existence of pure meromorphic integrals. Using the reduction map $E: \mathbf{M} \rightarrow U$, we define a holomorphic function $P$ into the Riemann sphere $\overline{\mathbf{C}}$ and show that $\mathbf{w}$ has a meromorphic integral if and only if the envelope of holomorphy of $P$ contains an open subset that is an analytic cover over M. Then, in preparation for $\S 4$, we prove that $\mathbf{w}$ has a meromorphic integral that separates all the integral curves if and only if the space $X_{w}$, obtained by identifying the points on the same integral curve, is a Hausdorff space.

We show in $\S 4$ that $\mathbf{w}$ is conjugate to a linear form $r y d x-s x d y$, where $r, s$ are positive integers, if and only if $X_{\mathbf{w}}$ is a Hausdorff space. Then we characterize the meromorphic functions that are conjugate to the rational functions $R\left(x^{r} / y^{s}\right)$, where $R$ is a rational function on $\overline{\mathbf{C}}$.

The last section deals with the nonexistence of transcendental integrals. We prove that

$$
\mathbf{W}=\left(x y-c y^{2}-c y^{3}\right) d x+\left(-x^{2}+c x y+2 c x y^{2}-y^{3}-y^{4}\right) d y,
$$

where

$$
c=\frac{\sqrt{2}+1}{\sqrt{2}-1},
$$

is a well-behaved differential form that does not admit a transcendental integral. Then an argument similar to that used by D. Cerveau and J. F. Mattei in [2] demonstrates the nonexistence of a topological criterion for transcendental integrability. 
The author wishes to express his deepest gratitude to his teacher, Professor F. Treves.

\section{Preliminaries}

1.1. Singular holomorphic foliations. Let $\mathbf{w}=a(x, y) d x+b(x, y) d y$ be a holomorphic differential form in a neighborhood $U$ of $0 \in \mathbf{C}^{2}$ and let $S_{\mathrm{w}}$ be the singular locus of $\mathbf{w}$; i.e., $S_{\mathbf{w}}$ is the analytic variety $\{p \in U ; \mathbf{w}(p)=0\}$. Then $\mathbf{w}$ defines a foliation $\mathbf{F}_{\mathbf{w}}$ of $U-S_{\mathbf{w}}$ : a leaf of $\mathbf{F}_{\mathbf{w}}$ through the point $p$ is the integral curve of $\mathbf{w}$, i.e., the orbit through $p$ of the differential equation

$$
\left\{\begin{array}{l}
\frac{d x}{d z}=-b(x, y), \\
\frac{d y}{d z}=a(x, y),
\end{array} \quad z \in C .\right.
$$

Since the coefficients of $\mathbf{w}$ are holomorphic functions, then the germ of $\mathbf{w}$ at 0 can be written as $\mathbf{w}=g \mathbf{v}$, where $g$ is the "greatest common divisor" of the coefficients $a, b$ and where $\mathbf{v}$ is a holomorphic differential form whose singular locus is contained in $\{0\}$. The singular foliation $\mathbf{F}_{\mathbf{v}}$, defined by $\mathbf{v}$, coincides with $\mathbf{F}_{\mathbf{w}}$ outside $S_{\mathbf{w}}$. This new foliation is called the saturate of $\mathbf{F}_{\mathbf{w}}$ and will be denoted by $\overline{\mathbf{F}}_{\mathbf{w}}$ (see [2]).

More generally, as in [1], we define a singular holomorphic foliation $\mathbf{F}$ on a 2-dimensional complex manifold $\mathbf{M}$ as the datum of

(a) a discrete subset of points $S(\mathbf{F})$ in $\mathbf{M}$ : the set of singularities of $\mathbf{F}$;

(b) an open covering $\left\{U_{j}\right\}_{j \in J}$ of $\mathbf{M}$ and a collection of holomorphic 1-forms $\left\{\mathbf{w}_{j}\right\}_{j \in J}$ that define $\mathbf{F}$. More precisely, the following conditions hold:

$\left(\mathbf{b}_{1}\right)$ The differential form $\mathbf{w}_{j}$, defined in $U_{j}$, vanishes exactly on $S(\mathbf{F}) \cap U_{j}$.

$\left(\mathbf{b}_{2}\right)$ The leaves of $\mathbf{F}$ in $U_{j}$ are the integral curves of $\mathbf{w}_{j}$.

$\left(\mathrm{b}_{3}\right)$ If $U_{j} \cap U_{i} \neq \varnothing$, then there is a holomorphic function $f_{j i}: U_{j} \cap U_{i} \rightarrow \mathbf{C}^{*}$ such that

$$
\mathbf{w}_{j}=f_{j i} \mathbf{w}_{i} .
$$

1.2. The blow-up of a point. We first recall the blow-up of $\mathbf{C}^{2}$ at 0 . Let $\mathbf{C} \mathbf{P}^{1}$ be the complex projective space of dimension 1 , that is, the set of complex lines in $\mathbf{C}^{2}$ that pass through the origin, and let $\mathbf{q}: \mathbf{C}^{2}-\{0\} \rightarrow \mathbf{C} \mathbf{P}^{1}$ be the quotient map: $\mathbf{q}(p)$ is the complex line in $\mathbf{C}^{2}$ through $p$ and the origin 0 .

Definition 1.21 [14]. The blow-up of $\mathbf{C}^{2}$ at 0 is the subset of $\mathbf{C}^{2} \times \mathbf{C P}^{1}$ given as

$$
\mathbf{C}_{0}^{2}=\left\{(p, \mathbf{q}(p)) \in\left(\mathbf{C}^{2}-\{0\}\right) \times \mathbf{C} \mathbf{P}^{1}\right\} \cup\left(\{0\} \times \mathbf{C P}^{1}\right) .
$$

Recall that $\mathbf{C}_{0}^{2}$ is a 2-dimensional complex manifold. More precisely if $V_{1}=$ $\mathbf{C} \mathbf{P}^{1}-\mathbf{q}((0,1)), V_{2}=\mathbf{C} \mathbf{P}^{1}-\mathbf{q}((1,0)), t: V_{1} \rightarrow \mathbf{C}, s: V_{2} \rightarrow \mathbf{C}$ are the canonical charts of $\mathbf{C P}^{1}$, i.e., $t=y / x$ and $s=x / y$, and if $P: \mathbf{C}_{0}^{2} \rightarrow \mathbf{C P}^{1}$ is the projection, then the maps $\phi_{1}: P^{-1}\left(V_{1}\right) \rightarrow \mathbf{C}^{2}$ and $\phi_{2}: P^{-1}\left(V_{2}\right) \rightarrow \mathbf{C}^{2}$, defined 
by

$$
\begin{array}{ll}
\phi_{1}((x, y), \mathbf{q}(x, y))=(x, y / x), & \phi_{1}(0, \mathbf{q}(x, y))=(0, y / x), \\
\phi_{2}((x, y), \mathbf{q}(x, y))=(x / y, y), & \phi_{2}(0, \mathbf{q}(x, y))=(x / y, 0),
\end{array}
$$

form an atlas of $\mathbf{C}_{0}^{2}$. The coordinates $(x, t),(s, y)$ of a point are related by

$$
y=t x \quad \text { and } \quad s=1 / t .
$$

The first projection $E_{0}: \mathbf{C}_{0}^{2} \rightarrow \mathbf{C}^{2}$ is called the blow-up map. It is defined in terms of the coordinates $(x, t),(s, y)$ by

$$
E_{0}(x, t)=(x, t x) \text { and } E_{0}(s, y)=(s y, y) .
$$

The map $E_{0}$ is a biholomorphism between $\mathbf{C}_{0}^{2}-E_{0}^{-1}(0)$ and $\mathbf{C}^{2}-\{0\}$, and the exceptional divisor $E_{0}^{-1}(0)=\{0\} \times \mathbf{C} \mathbf{P}^{1}$ will be identified with $\mathbf{C} \mathbf{P}^{1}$.

More generally, the blow-up of a 2-dimensional complex manifold $\mathbf{M}$ at a point $p$ consists in replacing $p$ by $\mathbf{C P}^{1}$. To do so, consider, as in [1], $T_{p} \mathbf{M}$ the tangent space of $\mathbf{M}$ at $p$ and let $\psi: U \rightarrow V$ be a biholomorphism between the open sets $U \subset \mathbf{M}$ and $V \subset T_{p} \mathbf{M}$ such that $p \in U$ and $\psi(p)=0$, and let $E_{0}:\left(T_{p} \mathbf{M}\right)_{0} \rightarrow T_{p} \mathbf{M}$ be the blow-up of $T_{p} \mathbf{M}$ at $0\left(T_{p} \mathbf{M}\right.$ is identified with $\left.\mathbf{C}^{2}\right)$. Then the mapping $E_{0}^{-1} \circ \psi$ is a biholomorphism between $U-\{p\}$ and $E_{0}^{-1}(V-\{0\})$. The blow-up $\mathbf{M}_{p}$ of $\mathbf{M}$ at $p$ is the 2-dimensional manifold

$$
(\mathbf{M}-\{p\}) \cup_{E_{0}^{-1} \circ \psi} E_{0}^{-1}(V),
$$

where $\cup_{E_{0}^{-1} \circ \psi}$ denotes the identification via $E_{0}^{-1} \circ \psi$ of the points in $U-\{p\}$ with the corresponding points in $E_{0}^{-1}(V-\{0\})$.

1.3. Desingularization of a germ of a differential form. Let $\mathbf{w}(x, y)=a(x, y) d x$ $+b(x, y) d y$ be the germ at $0 \in \mathbf{C}^{2}$ of a holomorphic differential form such that $\mathbf{w}(0)=0$ and 0 is the only singularity in a small neighborhood $U$ of 0 . Let

$$
\sum_{k=1}^{\infty}\left(a_{k}(x, y) d x+b_{k}(x, y) d y\right)
$$

be the Taylor expansion of $\mathbf{w}\left(a_{k}, b_{k}\right.$ are homogeneous polynomials of degree $k)$. The smallest integer $\nu$ such that $a_{\nu} d x+b_{\nu} d y$ is not identically zero is called the order of $\mathbf{w}$ at 0 . We will also refer to $\nu$ as the order at 0 of the foliation $\mathbf{F}_{\mathbf{w}}$. Write

$$
\mathbf{w}_{\nu}(x, y)=a_{\nu}(x, y) d x+b_{\nu}(x, y) d y
$$

and

$$
\mathbf{w}(x, y)=\left(a_{\nu}(x, y)+\alpha(x, y)\right) d x+\left(b_{\nu}(x, y)+\beta(x, y)\right) d y,
$$

where $\alpha, \beta$ are holomorphic functions of order $\geq \nu+1$ at 0 . Following the terminology in [2], the differential form $\mathbf{w}$ is said to be dicritic if the homogeneous polynomial

$$
P_{\nu+1}(x, y)=x a_{\nu}(x, y)+y b_{\nu}(x, y)
$$


is identically zero. Otherwise $\mathbf{w}$ is said to be nondicritic.

Let $E_{0}$ be the blow-up map of $\mathbf{C}^{2}$ at 0 . The pullback $E_{0}^{*} \mathbf{w}$ of $\mathbf{w}$ is a holomorphic differential form on $E_{0}^{-1}(U)$, whose expressions in the charts $(x, t),(s, y)$ are:

$$
\begin{aligned}
& E_{0}^{*} \mathbf{w}(x, t)=x^{\nu}\left(P_{\nu+1}(1, t)+x A(x, t)\right) d x+x^{\nu+1}\left(b_{\nu}(1, t)+\frac{(x, t x)}{x^{\nu}}\right) d t, \\
& E_{0}^{*} \mathbf{w}(s, y)=y^{\nu}\left(P_{\nu+1}(s, 1)+y B(s, y)\right) d y+y^{\nu+1}\left(a_{\nu}(s, 1)+\frac{(s y, y)}{y^{\nu}}\right) d s,
\end{aligned}
$$

where $P_{\nu+1}$ is the polynomial in (1.31) and where

$$
A(x, t)=\frac{(x, t x)+t(x, t x)}{x^{\nu}} \quad \text { and } \quad B(s, y)=\frac{s(s y, y)+(s y, y)}{y^{\nu}} .
$$

The saturated foliation $\mathbf{F}_{E_{0}^{*} \mathbf{w}}$ on $E_{0}^{-1}(U)$ is defined in the charts $(x, t),(s, y)$ by the differential forms

$$
\widetilde{\mathbf{w}}_{1}(x, t)=\frac{E_{0}^{*} \mathbf{w}(x, t x)}{x^{k}} \quad \text { and } \quad \widetilde{\mathbf{w}}_{2}(s, y)=\frac{E_{0}^{*} \mathbf{w}(s y, y)}{y^{k}},
$$

where $k=\nu+1$ in the dicritic case and $k=\nu$ otherwise. The singularities of $\overline{\mathbf{F}}_{E_{0}^{*} w}$ are on $\mathbf{C P}^{1}\left(=E_{0}^{-1}(0)\right)$ and are given in the nondicritic case by the equations

$$
P_{\nu+1}(1, t)=0, \quad P_{\nu+1}(s, 1)=0
$$

and in the dicritic case by the equations

$$
b_{\nu}(1, t)=A(0, t)=0, \quad a_{\nu}(s, 1)=B(s, 0)=0 .
$$

Note that when $\mathbf{w}$ is nondicritic, the complex curve $\mathbf{C} \mathbf{P}^{1}-\left\{p_{1}, \ldots, p_{k}\right\}$, where $\left\{p_{1}, \ldots, p_{k}\right\}$ is the set of singularities of $\overline{\mathbf{F}}_{E_{0}^{*} \mathbf{w}}$, is a leaf of $\overline{\mathbf{F}}_{E_{0}^{*} \mathbf{w}}$. When $\mathbf{w}$ is dicritic, the complex curve $\mathbf{C P}^{1}-\left(\left\{p_{1}, \ldots, p_{k}\right\} \cup\left\{q_{1}, \ldots, q_{m}\right\}\right)$, where $\left\{q_{1}, \ldots, q_{m}\right\}$ is the set of zeros of the polynomials $b_{\nu}(1, t)$ and $a_{\nu}(s, 1)$, is transversal to the foliation $\overline{\mathbf{F}}_{E_{0}^{*} \text { w }}$ and the leaves through $q_{1}, \ldots, q_{m}$ are tangent to $\mathbf{C P}^{1}$.

It is shown in [1, 2,6] and [10], for example, that the singularities of $\overline{\mathbf{F}}_{E_{0}^{*} \mathbf{w}}$ are simpler than that of $\mathbf{F}_{w}$; i.e., the order of the singularities diminish after blow-ups. Hence a series of blow-ups will produce a foliation whose singularities are reduced. More precisely

Definition 1.31 [2]. A holomorphic differential form $w$ on a two-dimensional complex manifold $\mathbf{M}$ is said to be reduced at $m \in \mathbf{M}$ if there are coordinates $(u, v)$ centered at $m$, a holomorphic function $h$ defined in a neighborhood of $m$ and a holomorphic differential form $\mathbf{v}$ such that $\mathbf{w}=h \mathbf{v}$ and either $\mathbf{v}(m) \neq 0$, or the first jet of $\mathbf{v}$ has one of the following forms:

(*) $u d v$, 
(**) $c_{1} v d u-c_{2} u d v$, with $c_{1} c_{2} \neq 0$ and $c_{1} / c_{2} \notin \mathbf{Q}^{+}$, where $\mathbf{Q}^{+}$denotes the set of positive rational numbers. When $\mathbf{w}$ is reduced at all the points of $\mathbf{M}$, we will say that $\mathbf{w}$ (or the saturate foliation $\overline{\mathbf{F}}_{\mathbf{w}}$ ) is reduced on $\mathbf{M}$.

Reduction Theorem 1.31 [2]. Let $\mathbf{w}$ be a holomorphic differential form in an open neighborhood $U$ of $0 \in \mathbf{C}^{2}$ and assume that 0 is the only singularity of $\mathbf{w}$. Then there is a proper holomorphic map $E: \mathbf{M} \rightarrow U$ from a 2-dimensional complex manifold $\mathbf{M}$ onto $U$, obtained by composition of blow-ups, such that:

(i) The exceptional divisor $E^{-1}(0)$ is a hypersurface with normal crossings;

(ii) The restriction of $E$ to $\mathbf{M}-E^{-1}(0)$ is a biholomorphism onto $U-\{0\}$;

(iii) The foliation $\overline{\mathbf{F}}_{E^{*}{ }_{\mathbf{w}}}$ is reduced on $\mathbf{M}$;

(iv) The branches of $E^{-1}(0)$ are either leaves or else transversal to the foliation $\overline{\mathbf{F}}_{E^{*} \mathbf{w}}$.

1.4. Integrals of a germ of a holomorphic differential form. An integral of a germ at $0 \in \mathbf{C}^{2}$ of a holomorphic differential form $\mathbf{w}$ is a germ at 0 of a "function" $f$ constant on the leaves of $\overline{\mathbf{F}}_{\mathbf{w}}$. When $d f$ is defined, we have $\mathbf{w} \wedge d f=0$. A precise definition of the various integrals is

Definition 1.41. (i) A germ at $0 \in \mathbf{C}^{2}$ of a holomorphic function $f$ is said to be an integral of $\mathbf{w}$ if

$$
\mathbf{w} \wedge d f=0
$$

in a neighborhood of 0 .

(ii) A germ at $0 \in \mathbf{C}^{2}$ of a pure meromorphic function $m=\alpha / \beta(\alpha, \beta$ germs of nonunit coprime holomorphic functions) is an integral of $w$ if

$$
(\alpha d \beta-\beta d \alpha) \wedge \mathbf{w}=0,
$$

or equivalently, $d m \wedge \mathbf{w}=0$ when $\beta \neq 0$ and $d(1 / m) \wedge \mathbf{w}=0$ when $\alpha \neq 0$.

(iii) A transcendental integral of $\mathbf{w}$ is a holomorphic function $T$ into $\overline{\mathbf{C}}$, defined on $U-\left(\gamma_{1} \cup \cdots \cup \gamma_{N}\right)$, where $U$ is an open neighborhood of 0 and $\gamma_{1}, \ldots, \gamma_{N}$ finitely many integral curves of $\mathbf{w}$, such that

$$
d T \wedge \mathbf{w}=0 \quad \text { in } U-\left(\gamma_{1} \cup \cdots \cup \gamma_{N}\right) .
$$

(iv) A multiform integral of $\mathbf{w}$ is a holomorphic function $\bar{H}$ into $\overline{\mathbf{C}}$, defined in the universal covering $\mathbf{U}$ of $U-\left(\gamma_{1} \cup \cdots \cup \gamma_{N}\right)$, where $\gamma_{1}, \ldots, \gamma_{N}$ are finitely many integral curves of $\mathbf{w}$, such that if $\mathbf{p}: \mathbf{U} \rightarrow U-\left(\gamma_{1} \cup \cdots \cup \gamma_{N}\right)$ is the covering map, then

$$
d \bar{H} \wedge \mathbf{p}^{*} \mathbf{w}=0 .
$$

When $\mathbf{w}$ has a holomorphic or a meromorphic integral in $U$, then the leaves of $\mathbf{F}_{\mathbf{w}}$ are the connected components in $U-\{0\}$ of the level sets of an integral. Hence, the closure in $U$ of the leaves are analytic varieties. Moreover, when $\mathbf{w}$ has a holomorphic integral, then only a finite number of integral curves adhere 
to $0\left(f^{-1}(f(0))\right.$ is an analytic variety, so it has a finite number of irreducible components). Conversely, J. F. Mattei and R. Moussu show the following theorem.

Theorem 1.41 [6]. Let $\mathbf{w}$ be the germ at $0 \in \mathbf{C}^{2}$ of a holomorphic differential form with an isolated singularity at 0 and such that

(i) all the leaves of $\mathbf{F}_{\mathbf{w}}$ are closed in $\mathbf{C}^{2}-\{0\}$, and

(ii) only a finite numbers of leaves accumulate at 0 .

Then $\mathbf{w}$ has a holomorphic integral.

Note that the condition (i) in Theorem 1.41 implies (by the Remmert-Stein removable singularity theorem, see [3] for example) that the closure of the leaves of $\mathbf{F}_{\mathbf{w}}$ are analytic varieties. A factorization result for integrals is also proved in [6].

Theorem 1.42 [6]. Let $f$ and $g$ be the germs at 0 of holomorphic integrals of $\mathbf{w}$ such that $f$ is not a power of a holomorphic function. Then there is a germ at $f(0) \in \mathbf{C}$ of a holomorphic function $h$ such that $g=h \circ f$.

Contrary to holomorphic integrability (Theorem 1.41), there is no topological criterion for meromorphic integrability (see [2] or $\S 5$ here). However, the following theorem in the meromorphic situation is an equivalent of Theorem 1.42 .

Theorem 1.43 [2]. Let $\mathbf{w}$ be the germ at $0 \in \mathbf{C}^{2}$ of a holomorphic differential form that admits a pure meromorphic integral. Then there is a germ of a meromorphic function $m_{0}$, unique up to a left composition by a Möbius transformation, such that if $m$ is a meromorphic integral of $\mathbf{w}$, then $m=R \circ m_{0}$, where $R$ is a rational function on the Riemann sphere $\mathbf{C}$.

The above theorems are in fact proved in $\mathbf{C}^{n}$; i.e., $\mathbf{w}$ is a germ at $0 \in \mathbf{C}^{n}$ of an integrable holomorphic differential form $(\mathbf{w} \wedge d \mathbf{w}=0)$. Also, it is proved in [2, 5 and 6] that if (1.41) (resp. (1.42)) is formally true, i.e., there are formal power series such that (1.41) (resp. (1.42)) holds, then $\mathbf{w}$ is holomorphically (resp. meromorphically) integrable.

\section{Desingularization AND INTEgRATING FACTOR OF WELL-BEHAVED DIFFERENTIAL FORMS}

Next to the foliations that are given by the level sets of a holomorphic function, the simplest ones are those for which the closure of the leaves are analytic varieties that pass through 0 . We will say that the germ at $0 \in \mathbf{C}^{2}$ of a holomorphic differential form is well behaved if the closure in a neighborhood of 0 of the integral curves are analytic varieties that pass through 0 . An example of well-behaved foliation is furnished by the level sets of a pure meromorphic function.

We prove in this section, as a consequence of the reduction Theorem 1.31 and a linearization theorem for reduced forms [6], that if $\mathbf{w}$ is well behaved, then 
the singularities of $\overline{\mathbf{F}}_{E^{*} \text { w }}(E: \mathbf{M} \rightarrow U$ is the reduction map of Theorem 1.31) are of the form $p v d u+q u d v$, where $(u, v)$ are the appropriate coordinates in Theorem 1.31 and $p, q$ are positive integers. We then show that the existence of an integrating factor for well-behaved differential forms can be reduced into solving a kind of Cousin problem in the unit disc. But first recall a linearization theorem for reduced forms, proved by J. F. Mattei and R. Moussu.

Theorem 2.11 [6]. Let $\mathbf{w}$ be the germ at $0 \in \mathbf{C}^{2}$ of a reduced differential form. Then the following properties are equivalent:

(i) There is a biholomorphism $\phi:\left(\mathbf{C}^{2}, 0\right) \rightarrow\left(\mathbf{C}^{2}, 0\right)$, a holomorphic function $g$ and two positive integers $p, q$ such that

$$
\phi^{*} \mathbf{w}(x, y)=g(x, y)(p y d x+q x d y) ;
$$

(ii) $\mathbf{w}$ has a holomorphic integral;

(iii) the integral curves of $\mathbf{w}$ are closed in $\mathbf{C}^{2}-\{0\}$.

The reduction theorem for well-behaved differential forms is

Theorem 2.12. Let $\mathbf{w}$ be the germ at $0 \in \mathbf{C}^{2}$ of a well-behaved differential form, $E: \mathbf{M} \rightarrow U$ the reduction map of Theorem 1.31 , and $m \in E^{-1}(0)$ a singularity of $\overline{\mathbf{F}}_{E^{*}{ }_{w}}$. Then there are coordinates $(u, v)$ centered at $m$, a holomorphic function $g$ and two positive integers $p, q$ such that:

$$
E^{*} \mathbf{w}(u, v)=g(u, v)(p v d u+q u d v) .
$$

Moreover, a branch of the exceptional divisor $E^{-1}(0)$ is transversal to the foliation $\overline{\mathbf{F}}_{E^{*} w^{*}}$.

The proof of this theorem is not difficult. However, to give a complete description of the situation, we first prove some elementary lemmas. Let us start by recalling what is meant by a reduced form to be stable by blow-up.

Lemma 2.11. Let $\mathbf{w}$ be the germ at $0 \in \mathbf{C}^{2}$ of a reduced differential form. Then $\overline{\mathbf{F}}_{E_{0}^{*} \text { w }}$ is reduced $\left(E_{0}: \mathbf{C}_{0}^{2} \rightarrow \mathbf{C}^{2}\right.$ is the blow-up at 0$)$.

Proof. Write

$$
\mathbf{w}(x, y)=\left(c_{1} y+\alpha(x, y)\right) d x+\left(-c_{2} x+\beta(x, y)\right) d y,
$$

where $c_{1}, c_{2} \in \mathbf{C}$ are such that $c_{1} c_{2} \neq 0$ and $c_{1} / c_{2} \notin \mathbf{Q}^{+}$or $c_{1}=0, c_{2} \neq 0$ and where $\alpha, \beta$ are holomorphic functions with order $\geq 2$ at 0 . Then in the chart $(x, t)$ of $\mathbf{C}_{0}^{2}$ the foliation $\overline{\mathbf{F}}_{E_{0}^{*} \mathbf{w}}$ is defined by

$$
\overline{\mathbf{w}}_{1}(x, t)=\left(\left(c_{1}-c_{2}\right) t+\frac{\alpha(x, t x)+t \beta(x, t x)}{x}\right) d x+\left(-c_{2} x+\beta(x, t x)\right) d x .
$$

The only singularity of $\overline{\mathbf{w}}_{1}$ on the complex curve $\{x=0\}$ is the point $(x=$ $0, t=0)$. To show that this singularity is reduced, we have just to remark that if

$$
\rho(x, t)=\frac{\alpha(x, t x)+t \beta(x, t x)}{x^{2}}
$$


and $A=\rho(0,0)$, then the change of variables

$$
x=x^{\prime}, \quad t=\frac{-A}{c_{1}-2 c_{2}} x^{\prime}+t^{\prime}
$$

transforms $\overline{\mathbf{w}}_{1}$ into

$$
\overline{\mathbf{w}}_{1}\left(x^{\prime}, t^{\prime}\right)=\left(c_{1}-c_{2}\right) t^{\prime} d x^{\prime}-c_{2} x^{\prime} d t^{\prime}+\eta,
$$

where $\eta$ is a differential form with order $\geq 2$ at $\left(x^{\prime}=0, t^{\prime}=0\right)$. A similar argument shows that in the chart $(s, y)$ of $\bar{C}_{0}^{2}$ the only singularity $(s=0, y=$ 0 ) of $\overline{\mathbf{F}}_{E_{0}^{*} \mathbf{w}}$ is also reduced. The lemma is there proved.

In order to continue, we need to recall the notion of the tangent cone of a holomorphic function at a point. Let $f(x, y)$ be the germ at $0 \in \mathbf{C}^{2}$ of holomorphic function of order $\nu$ and let $\bar{f}_{1}\left(\right.$ resp. $\left.\bar{f}_{2}\right)$ be the holomorphic function defined in the chart $(x, t)$ (resp. $(s, y))$ of $\mathbf{C}_{0}^{2}$ by

$$
\bar{f}_{1}(x, t)=\frac{f(x, t x)}{x^{\nu}} \quad\left(\operatorname{resp} . \bar{f}_{2}(s, y)=\frac{f(s y, y)}{y^{\nu}}\right) .
$$

In the intersection of the charts $(x, t),(s, y)$ (when $t \neq 0$ and $s \neq 0$ ), the functions $\bar{f}_{1}, \bar{f}_{2}$ are related by

$$
\bar{f}_{1}(x, t)=t^{\nu} \bar{f}_{2}\left(\frac{1}{t}, x t\right) .
$$

So, outside $\{t=0\} \cup\{s=0\}$ the functions $\bar{f}_{1}$ and $\bar{f}_{2}$ have the same zeros. The set $C(f, 0)=\left\{p \in E_{0}^{-1}(0) ; f_{1}(p)=0\right.$ or $\left.f_{2}(p)=0\right\}$ is called the tangent cone of $f$ at 0 . The following lemma (proved in a more general context in [14]) relates the irreducibility of $f$ to that of $C(f, 0)$.

Lemma 2.12. If $f$ is irreducible, then $C(f, 0)$ is reduced to a point.

In the next lemma we prove that if the sequence obtained by successive blowups of the tangent cones of an analytic variety are all contained in the north and south poles of $\mathbf{C P}^{1}$, then this variety is either the $x$-axis or either the $y$-axis. Let us first introduce some notation. Let $f$ be the germ at $0 \in \mathbf{C}^{2}$ of an irreducible holomorphic function. Denote by $p_{0}$ the tangent cone $C(f, 0)$. Let $f_{0}$ be the defining function of the irreducible variety $V_{0}=\mathrm{Cl} E_{0}^{-1}(V-\{0\})$, where $\mathrm{Cl}$ denotes the closure and $V$ the variety $\{f=0\}$. Note that $\bar{f}_{1}$ or $\bar{f}_{2}$ or both if $p_{0} \neq(x=0, t=0)$ and $p_{0} \neq(s=0, y=0)$ vanish on $V_{0}=\left\{f_{0}=0\right\}$. Let $E_{1}:\left(\mathbf{C}_{0}^{2}\right)_{p_{0}} \rightarrow \mathbf{C}_{0}^{2}$ be the blow-up map of $\mathbf{C}_{0}^{2}$ at $p_{0}$. Denote by $p_{1}$ the tangent cone $C\left(f_{0}, p_{0}\right)$ and by $f_{1}$ a defining function of $V_{1}=\mathrm{Cl} E_{1}^{-1}\left(V_{0}-\left\{p_{0}\right\}\right)$. More generally, we define $f_{i}, p_{i}$ by $p_{i}=C\left(f_{i-1}, p_{i-1}\right)$ and $f_{i}$ a defining function of $V_{i}=\mathrm{Cl} E_{i}^{-1}\left(V_{i-1}-\left\{p_{i-1}\right\}\right)$. Finally, the north and south poles of $\mathbf{C P}^{1}$ will be denoted by $N$ and $S$, i.e., $N=(x=0, t=0)$ and $S=(s=0, y=0)$. 
Lemma 2.13. Let $f$ be the germ at $0 \in \mathbf{C}^{2}$ of an irreducible holomorphic function such that $p_{i} \in\{N, S\}$ for every $i \in \mathbf{Z}^{+}$. Then, up to units,

$$
f(x, y)=x \quad \text { or } \quad f(x, y)=y .
$$

Proof. We distinguish first the case where $f$ has order 1 at 0 . Hence, we can assume $\partial f(0) / \partial y=1$. If in addition $\partial^{n} f(0) / \partial x^{n}=0$ for every $n \in \mathbf{Z}^{+}$, then $f(x, y)=y g(x, y)$, where $g$ is a nonvanishing holomorphic function and the lemma is proved in this situation. Actually, the other eventuality cannot occur. Indeed, if

$$
f(x, y)=y+a_{n} x^{n}+x^{n+1} \alpha_{1}(x)+y^{2} \alpha_{2}(y)+x y \alpha_{3}(x, y),
$$

where $a_{n} \in \mathbf{C}^{*}, \alpha_{1}, \alpha_{2}$ and $\alpha_{3}$ are holomorphic functions, then

$$
f_{0}(x, t)=t+a_{n} x^{n-1}+x^{n} \alpha_{1}(x)+t^{2} x \alpha_{2}(x t)+x t \alpha_{3}(x, t x) .
$$

So after $(n-1)$ blow-ups, we obtain

$$
f_{n-1}\left(x, t_{n-1}\right)=t_{n-1}+a_{n} x+g\left(x, t_{n-1}\right),
$$

where $t_{n-1}$ is a coordinate in the corresponding branch and $g$ a holomorphic function of order $\geq 2$ at $\left(x=0, t_{n-1}=0\right)$. But then

$$
C\left(f_{n-1}, 0\right)=\left\{\left(x=0, t_{n}=-a_{n}\right)\right\} \not \subset\{N, S\} ;
$$

i.e., the hypothesis of the lemma is violated. To complete the proof of the lemma, we have to remark that the other case also cannot occur. For, if the order of $f$ at 0 were $\geq 2$, then after a finite number of blow-ups, we would obtain an $f_{n}$ with order 1 (reduction of singularities of a complex curve) and the previous case applies to $f_{n}$. Hence, $V=\{f=0\}$ is one of the axes; i.e.,

$$
f(x, y)=x^{k} g(x, y) \text { or } f(x, y)=y^{k} g(x, y)
$$

with $g(0,0) \neq 0$ and $k \geq 2$. This is impossible because of the irreducibility of $f$.

Corollary 2.11. Let $\mathbf{w}$ be the germ at $0 \in \mathbf{C}^{2}$ of a reduced differential form and let $\gamma$ be an integral curve whose closure is an analytic variety that passes through 0 . Then $\gamma$ is one of the axes.

Proof. The tangent cone of the closure of $\gamma$ is contained in the singularities $\{N, S\}$ of the reduced foliation $\overline{\mathbf{F}}_{E_{0}^{*} \mathbf{w}}$ (see Lemma 2.11). By continuing this processes, we see that the hypotheses of Lemma 2.13 are fulfilled and the conclusion follows.

Proof of Theorem 2.12. Let $E: \mathbf{M} \rightarrow U$ be the reduction map of Theorem 1.31 and let $m \in E^{-1}(0)$ be a (reduced) singularity of $\overline{\mathbf{F}}_{E^{*} \mathbf{w}}$. Since $\mathbf{w}$ is well behaved, then the closure of the leaves of $\overline{\mathbf{F}}_{E^{*}{ }^{*}}$ are analytic varieties that pass through $E^{-1}(0)$. Moreover, it follows from Corollary 2.11 that the leaves that accumulate to $m$ are the $u$ and $v$ axes (in the coordinate system $(u, v)$ of Theorem 1.31). Hence, only 2 leaves of $\overline{\mathbf{F}}_{E^{*} \mathbf{w}}$ accumulate to each one of its 
singularities. Therefore, the first part of the theorem follows from Theorem 2.11. To finish the proof, notice that the branches of $E^{-1}(0)$ are either leaves or transversal to the foliation $\overline{\mathbf{F}}_{E^{*} w}$. If all the branches were leaves, then only a finite number of leaves of $\overline{\mathbf{F}}_{E^{*}{ }_{w}}$ would accumulate to its finite number of singularities.

A holomorphic (resp. meromorphic) integrating factor of a differential form $\mathbf{w}$ is a holomorphic (resp. meromorphic) function $h$ such that $\mathbf{w} / h$ is a closed form. It is proved in [2] (Theorem 2.1, p. 37) that if $\mathbf{w}$ has an integrating factor, then it has a multiform integral. We associated to a well-behaved form a kind of a Cousin problem, called here problem $\mathrm{P}$. The solvability of this problem insures the existence of an integrating factor.

Let $D$ be the unit disc in $\mathbf{C}$ and $\left\{\left(V_{i j}, \beta_{i j}\right)\right\}_{i, j \in J}$ a finite collection of simply connected subsets $V_{i j}$ of $D$ and holomorphic functions $\beta_{i j}$ satisfying the following:

(i) $\beta_{i j}: V_{i j} \rightarrow V_{j i}$ is a biholomorphism,

(ii) $\beta_{i j} \circ \beta_{j i}=\mathrm{id}$,

(iii) $\beta_{i j} \circ \beta_{j k} \circ \beta_{k i}=\mathrm{id}$.

The problem is the following:

Problem P. Find nontrivial holomorphic functions $l_{1}$ defined in $D$ such that

$$
l_{i}(z)=\beta_{i j}^{\prime}(z)\left(l_{j} \circ \beta_{i j}\right)(z) \quad \text { for all } i, j \in J \text { and } z \in V_{i j} .
$$

When problem $\mathrm{P}$ has a solution, we will refer to $\left(V_{i j}, \beta_{i j}\right)_{i, j}$ as admissible data.

Let $\mathbf{w}$ be a well-behaved differential form at $0 \in \mathbf{C}^{2}$ and $E: \mathbf{M} \rightarrow U$ be the reduction map of Theorem 1.31. Choose a finite covering $\left(\mathbf{O}_{i}\right)_{i}$ of a neighborhood of $E^{-1}(0) \subset \mathbf{M}$ such that in each $\mathbf{O}_{i}$ the foliation $\overline{\mathbf{F}}_{E^{*}{ }_{w}}$ has at most one singularity (of the type $p v d u+q u d v$, by Theorem 2.12) and $E^{*} \mathbf{w}$ has a holomorphic integral $h_{i}$ defined in $\mathbf{O}_{i}$ with the following properties:

(a) $h_{i}\left(\mathbf{O}_{i}\right)=D$,

(b) $V_{i j}=h_{i}\left(\mathbf{O}_{i} \cap O_{j}\right)$ is simple connected,

(c) $d h_{i}(m) \neq 0$ for every $m \in \mathbf{O}_{i} \cap \mathbf{O}_{j}$.

When $\mathbf{O}_{i} \cap \mathbf{O}_{j} \neq \varnothing$, the integrals $h_{i}$ and $h_{j}$ are related by

$$
h_{j}(m)=\beta_{i j} \circ h_{i}(m), \quad m \in \mathbf{O}_{i} \cap \mathbf{O}_{j},
$$

where $\beta_{i j}$ is a holomorphic function defined in $V_{i j}$. It follows from the choice of $\mathbf{O}_{i}$ and $h_{i}$ that $\left(V_{i j}, \beta_{i j}\right)$ are data for problem P. We show the following: if $\left(V_{i j}, \beta_{i j}\right)$ are admissible data for problem $\mathrm{P}$, then $\mathbf{w}$ has an integrating factor. For it follows from the fact that $h_{i}$ is an integral of $E^{*} \mathbf{w}$ in $\mathbf{O}_{i}$ that there is a holomorphic function $f_{i}$, defined in $\mathbf{O}_{i}$, such that

$$
\frac{E^{*} \mathbf{w}}{f_{i}}=d h_{i} \text {. }
$$


Also, since $h_{i}, h_{j}$ are related by $(2.21)$, then $f_{i}, f_{j}$ are related by

$$
f_{j}=\left(\beta_{i j} \circ h_{i}\right) f_{i} \quad \text { in } \mathbf{O}_{i} \cap \mathbf{O}_{j} \text {. }
$$

Next, define the holomorphic integrals $g_{i}$ of $E^{*} \mathbf{w}$ in $\mathbf{O}_{i}$ by

$$
g_{i}=l_{i} \circ h_{i},
$$

where the functions $l_{i}$ are the solutions of the problem $\mathrm{P}$ for the data $\left(V_{i j}, \beta_{i j}\right)$. Now, the mapping $F$ defined in $\mathbf{O}_{i}$ by

$$
F=g_{i} / f_{i}
$$

is a well-defined integrating factor of $E^{*} \mathbf{w}$ in a neighborhood of $E^{-1}(0) \subset \mathbf{M}$. To see this, write

$$
g_{i} / f_{i}=\left(l_{i} \circ h_{i}\right) / f_{i}=\beta_{i j}^{\prime}\left(l_{j} \circ \beta_{i j} \circ h_{i}\right) / f_{i}=\left(l_{j} \circ h_{j}\right) / f_{j}=g_{j} / f_{j}
$$

and

$$
d\left(F E^{*} \mathbf{w}\right)=g_{i} d\left(E^{*} \mathbf{w} / f_{i}\right)+\left(l / f_{i}\right) d g_{i} \wedge E^{*} \mathbf{w}=0 .
$$

Finally, $m(x, y)=F \circ E^{-1}(x, y)$, a holomorphic function into $\overline{\mathbf{C}}$ defined in $\mathbf{C}^{2}-\{0\}$, extends by E. E. Levi's theorem (see [7, p. 133]) as a meromorphic function to a neighborhood of 0 and clearly $m$ is an integrating factor of $\mathbf{w}$.

\section{MEROMORPHIC INTEGRALS}

We give here a necessary and sufficient condition for a well-behaved differential form to admit a pure meromorphic integral. Unfortunately, we do not have a criterion to check if an arbitrary differential form satisfies this condition. Then, in view of application in the next section, we give a necessary and sufficient condition for the existence of a meromorphic integral separating the integral curves.

First recall the notion of analytic cover (see [3]). We say that the triple $(\mathbf{R}, \mathbf{p}, \mathbf{M})$ is an analytic cover if

(i) $\mathbf{R}$ is a locally compact Hausdorff space.

(ii) $\mathbf{M}$ is a complex manifold.

(iii) $\mathbf{P}$ is a proper, light $\left(\mathbf{p}^{-1}(x)\right.$ consists of a discrete set of points for all $x \in \mathbf{M}$ ), continuous mapping of $\mathbf{R}$ onto $\mathbf{M}$.

(iv) There is a negligible set $A \subset \mathbf{M}$, and an integer $n$, such that $\mathbf{P}$ is an $n$-sheeted covering from $\mathbf{R}-\mathbf{p}^{-1}(A)$ onto $\mathbf{M}-A$.

(v) $\mathbf{R}-\mathbf{p}^{-1}(A)$ is dense in $\mathbf{R}$.

Let $\mathbf{w}$ be the germ at $0 \in \mathbf{C}^{2}$ of a well-behaved differential form and let $E: \mathbf{M} \rightarrow U$ be the reduction map. Let $\Sigma$ be a branch of $E^{-1}(0)$, transversal to the foliation $\mathbf{F}_{E^{*} \mathbf{w}}$ (the existence of such branches is guaranteed by Theorem $2.12)$. Let $\left\{p_{1}, \ldots, p_{N}\right\}$ be the subset of $\Sigma$ consisting of the singularities of $\mathbf{F}_{E^{*}{ }^{*} \text { }}$ and of the points through which the leaves are tangent to $\Sigma$. Let $\mathbf{O}$ be a neighborhood of $\Sigma-\left\{p_{1}, \ldots, p_{N}\right\}$ on which

$$
\#\left(\gamma_{p} \cap \Sigma\right)=1 \quad \text { for every } p \in \mathbf{O} \text {, }
$$


where $\gamma_{p}$ denotes the connected component through $p$ of the leaf of $\mathbf{F}_{E^{*} \mathbf{w}}$. Then define a function $P: \mathbf{O} \rightarrow \overline{\mathbf{C}}$ by

$$
P(p)=\gamma_{p} \cap \Sigma,
$$

where $\Sigma$ is identified with $\overline{\mathbf{C}}$. Clearly $P$ is an integral of $E^{*} \mathbf{w}$ in $\mathbf{O}$. Denote by $\mathbf{E}$ the envelope of holomorphy of $P$ and by $\mathbf{p}$ the corresponding projection into $\mathbf{M}$. We have the following theorem:

Theorem 3.1. Let $\mathbf{w}$ and $P$ be as above. Then $\mathbf{w}$ has a meromorphic integral if and only if the envelope of holomorphy $\mathbf{E}$ of $P$ contains an open subset $\mathbf{R}$ such that $(\mathbf{R}, \mathbf{p}, \mathbf{M})$ is an analytic cover.

Proof. Let $\Sigma, p_{1}, \ldots, p_{N}$ be as above and define, in a neighborhood $W_{i} \subset \Sigma$ of $p_{i}$, the equivalence relation $\sim$

$$
\zeta \sim \zeta^{\prime} \quad \text { if and only if } \quad \Gamma_{\zeta}=\Gamma_{\zeta^{\prime}} \quad \zeta, \zeta^{\prime} \in W_{i},
$$

where $\Gamma_{\zeta}$ is the leaf through $\zeta$ of $\mathbf{F}_{E^{*} \mathbf{w}}$. Consider a holomorphic integral $h_{i}$ of $E^{*} \mathbf{w}$ in a neighborhood of $p_{i} \in \mathbf{M}$ and let $\rho_{i}$ be its restriction to $\Sigma$. Denote by $f$ the germ at $p_{i} \in \Sigma$ of an invariance function of $\rho_{i}$, i.e., $f$ is such that

$$
\rho_{i} \circ f(\zeta)=\rho_{i}(\zeta) \text { for every } \zeta \text { near } p_{i} \text {. }
$$

Since the germ of an invariance function of a holomorphic function is conjugate to a rational rotation (see [6] for example) and

$$
\rho_{i}(\zeta)=\rho_{i}\left(\zeta^{\prime}\right) \quad \text { if } \zeta \sim \zeta^{\prime}
$$

( $h_{i}$ is constant on the leaves), then the equivalence relation $\sim$ is conjugate to a rational rotation; that is, there is an integer $k_{i}$ and a holomorphic function $\delta_{i}$, defined in a neighborhood of $p_{i} \in \Sigma$ such that

$$
\delta_{i}\left(k_{i}\right)=\underbrace{\delta_{i} \circ \cdots \circ \delta_{i}}_{k_{i} \text { times }}=\mathrm{id}
$$

and

$$
\zeta \sim \zeta^{\prime} \quad \text { if and only if } \quad \zeta^{\prime}=\delta_{i}^{(j)}(\zeta) \text { for some integer } j .
$$

Denote by $\theta_{1}, \ldots, \theta_{N}$ the global analytic continuations of $\delta_{1}, \ldots, \delta_{N}$ respectively.

Now, assume that $\mathbf{w}$ has a pure meromorphic integral $m(x, y)$. Then the function $V: \mathbf{M} \rightarrow \overline{\mathbf{C}}$ defined by

$$
V(p)=m \circ E(m) \quad \text { if } p \in \mathbf{M}-E^{-1}(0),
$$

constant on the leaves of $\mathbf{F}_{E^{*} w}$, extends to $E^{-1}(0)$ as a meromorphic integral of $E^{*} \mathbf{w}$. The restriction $R$ of $V$ to $\Sigma(=\overline{\mathbf{C}})$ is a rational function and the roots of the equation

$$
R(\zeta)=R\left(\zeta^{\prime}\right)
$$


are algebraic functions that form a finite group $G$ under the law of composition of functions. To see this, let $g(\zeta)$ and $l(\zeta)$ be two solutions of (3.2) and write

$$
R(\log (\zeta))=R(l(g(\zeta)))=R(g(\zeta))=R(\zeta) .
$$

Moreover, it follows from (3.1) and (3.2) that the functions $\theta_{1}, \ldots, \theta_{N}$ are algebraic functions and generate a finite subgroup $\left\langle\theta_{1}, \ldots, \theta_{N}\right\rangle$ of $G$. Hence, if $\widetilde{P}$ is the holomorphic extension of $P$ to a neighborhood of $\Sigma$, then its restriction $\alpha$ to $\Sigma$ is an algebraic function with branches id, $\alpha_{1}, \ldots, \alpha_{k}$. Define the rational function $R_{1}$ by

$$
R_{1}(\zeta)=\zeta \alpha_{1}(\zeta) \cdots \alpha_{k}(\zeta)
$$

and the compact Riemann surface

$$
S=\overline{\mathbf{C}} / R_{1}=\overline{\mathbf{C}} /\left\langle\theta_{1}, \ldots, \theta_{N}\right\rangle,
$$

obtained by identifying $\zeta \in \overline{\mathbf{C}}$ with its $\left\langle\theta_{1}, \ldots, \theta_{N}\right\rangle$-orbit (or equivalently, by identifying $\zeta$ with $\zeta^{\prime}$ s.t. $\left.R_{1}(\zeta)=R_{1}\left(\zeta^{\prime}\right)\right)$. $S$ is then an analytic cover over the compact Riemann surface

$$
S^{\prime}=\mathbf{C} / R=\mathbf{C} / G
$$

So,

$$
R(\zeta)=Q \circ R_{1}(\zeta)
$$

for some rational function $Q$. Therefore, the algebraic function $R_{1}^{-1} \circ Q^{-1} \circ R$ extends to $\mathbf{M}$ by

$$
\Phi(p)=R_{1}^{-1} \circ Q^{-1} \circ V(p)
$$

as a continuation of $P$ and its envelope of holomorphy is an analytic cover over M.

Conversely, assume that $(\mathbf{R}, \mathbf{p}, \mathbf{M})$ is an analytic cover and denote by $\widehat{P}$ the lift of $P(\widehat{P}: \mathbf{R} \rightarrow \overline{\mathbf{C}}$ is uniform $)$ and by $\mathbf{q}_{1}, \ldots, \mathbf{q}_{N}$ the $N$-branches of $\mathbf{p}^{-1}: \mathbf{M} \rightarrow \mathbf{R}$. Then the mapping

$$
\psi(p)=\left(\widehat{P} \circ q_{1}\right)(p) \cdots\left(\widehat{P} \circ q_{N}\right)(p)
$$

is a well-defined integral of $E^{*} \mathbf{w}$ in $\mathbf{M}$. Hence, the mapping

$$
m(x, y)=\psi \circ E^{-1}(x, y)
$$

is a meromorphic integral of $\mathbf{w}$ at $0 \in \mathbf{C}^{2}$. The theorem is therefore proved.

Define the equivalence relation $\sim$, on $U-\{0\}$, where $U$ is a small neighborhood of 0 , by

$$
p \sim p^{\prime} \quad \text { if and only if } \quad \gamma_{p}=\gamma_{p^{\prime}} .
$$

Here $\gamma_{p}$ denotes the integral curve through $p$ of $\mathbf{w}$. We equip the orbit space

$$
X_{\mathbf{w}}=(U-\{0\}) / \sim
$$

with the quotient topology and adopt the notation $\mathbf{r}: U-\{0\} \rightarrow X_{\mathbf{w}}$ for the quotient map and $\bar{p}=\mathbf{r}(p)$. We obtain the following proposition. 
Proposition 3.1. w has a pure meromorphic integral $m$ such that $m^{-1}(m(x))$ is irreducible for all $x \in U-\{0\}$ if and only if $X_{\mathrm{w}}$ is a Hausdorff space.

Proof of necessity. First recall, from [4] for example, that the germ of a pure meromorphic function assumes all the values of $\overline{\mathbf{C}}$. Hence, if $\mathbf{w}$ has a pure meromorphic integral $m$ such that $m^{-1}(m(x))$ is an irreducible variety for every $x \in U-\{0\}$, then $X_{\mathbf{w}}$ is homeomorphic to $m(U-\{0\})=\overline{\mathbf{C}}$.

The proof of the sufficiency is based upon two lemmas. But first introduce the following: let $T_{p}$ be a small transversal (complex) curve to $\gamma_{p}$ through the point $p$. Denote by $\widetilde{T}_{p}$ the quotient space of $T_{p}$ by the equivalence relation $\sim$ restricted to $T_{p}$. Then clearly $\widetilde{T}_{p}$ is homeomorphic to the open neighborhood $\mathbf{r}\left(T_{p}\right)$ of $p$ in $X_{\mathbf{w}}$. On the other hand, consider the analytic space $D_{n}$, obtained as the quotient of the unit disc $D$ by the function $z^{n}$ (two points $z, z^{\prime}$ are equivalent if $z^{n}=z^{\prime n}$ ). The first lemma is

Lemma 3.1. For every $\bar{p} \in X_{\mathbf{w}}$, there is an integer $n$ such that $\mathbf{r}\left(T_{p}\right)$ is homeomorphic to $D_{n}$. Moreover, only a finite number of points $p_{1}, \ldots, p_{N}$ are such that $\mathbf{r}\left(T_{p_{i}}\right)$ is homeomorphic to $D_{n_{i}}$ with $n_{i} \geq 2$.

Proof. Denote by $E: \mathbf{M} \rightarrow U$ the reduction map of Theorem 1.31 and by $L_{E}-1_{(p)}$ the leaf through the point $E^{-1}(p)$ of $\overline{\mathbf{F}}_{E^{*}{ }_{w}}$. We can select an open neighborhood $U$ of $0 \in \mathbf{C}^{2}$ in such a way that $\mathrm{Cl}\left(L_{E}-1_{(p)}\right) \cap E^{-1}(0)$ consists of one point $p_{0}\left(\mathrm{Cl}\right.$ denotes the closure). If $p_{0}$ is not a singularity of $\mathbf{F}_{E^{*} \mathbf{w}}$, then $E^{*} \mathbf{w}$ has an integral $h_{p_{0}}$ near $p_{0}$ such that its restriction $\rho_{p_{0}}$ to any small transversal curve to $L_{p_{0}}$ through $p_{0}$ satisfies

$$
d \rho_{p_{0}}\left(p_{0}\right) \neq 0 .
$$

That is, $\mathbf{r}\left(T_{p}\right)$ is homeomorphic to $D, n=1$ in this case. When $p_{0}$ is a singularity of $\overline{\mathbf{F}}_{E^{*}{ }_{\boldsymbol{w}}}$, then from Theorem 2.12 follows the existence of coordinates $(u, v)$ centered at $p_{0}$ on which $L_{E}-1_{(p)}$ is one of the axes and $u^{r} v^{s}$, for some coprime positive integers $r$ and $s$, is an integral of $E^{*} \mathbf{w}$. The quotient $\mathbf{r}\left(T_{p}\right)$, in this situation is homeomorphic to $D_{r}$ or $D_{s}$. To conclude the proof of the lemma, note that when $\mathbf{r}\left(T_{p}\right)$ is homeomorphic to $D_{n}$ with $n \geq 2$, then $L_{E}-1_{(p)}$ accumulates to a singularity of $\overline{\mathbf{F}}_{E^{*} w}$. Since the number of such singularities is finite and only two leaves adhere to each singularity, the conclusion follows.

Denote by $S$ the set $X_{\mathbf{w}}-\left\{p_{1}, \ldots, p_{N}\right\}$, where $p_{1}, \ldots, p_{N}$ are the points in Lemma 3.1. The second lemma is

Lemma 3.2. $S$ is a schlicht Riemann surface.

Proof. A chart about $\bar{p} \in S$ is given as

$$
\left(\mathbf{r}\left(T_{p}\right), \Phi_{p}^{-1} \circ \mathbf{r}^{-1}\right),
$$


where $\Phi_{p}: D \rightarrow T_{p}$ is a conformal map and where $\mathbf{r}^{-1}: \mathbf{r}\left(T_{p}\right) \rightarrow T_{p}$ means here a section of $\mathbf{r}$. The transition functions between two charts $\left(\mathbf{r}\left(T_{p}\right), \Phi_{p}^{-1} \circ \mathbf{r}^{-1}\right)$ and $\left(\mathbf{r}\left(T_{q}\right), \Phi_{q}^{-1} \circ \mathbf{r}^{-1}\right)$ are given on

$$
O_{p q}=\Phi_{p}^{-1}\left(T_{p} \cap\left(\bigcup_{x \in T_{q}} \gamma_{x}\right) \quad \text { and } \quad O_{q p}=\Phi_{q}^{-1}\left(T_{q} \cap\left(\bigcup_{x \in T_{p}} \gamma_{x}\right)\right)\right.
$$

respectively by

$$
\Phi_{q}^{-1} \circ \Phi_{p} \text { and } \Phi_{p}^{-1} \circ \Phi_{q} .
$$

This shows that $S$ is a complex manifold. To prove that $S$ is schlicht (a simple closed curve separates $S$ ), take $\Gamma$ a simple closed real analytic curve in $S$ and denote by $A$ its pre-image via $\mathbf{r}$ in $U$. Then $\operatorname{Cl}\left(E^{-1}(A)\right) \cap E^{-1}(0)$ is a simple real analytic curve in $E^{-1}(0)$ and so $\mathrm{Cl}\left(E^{-1}(A)\right)$ is a 3-dimensional real analytic manifold in $\mathbf{M}$. Therefore, its blow-down $\mathrm{Cl}(A)$, a 3-dimensional real analytic variety through $0 \in \mathbf{C}^{2}$, separates $U$. Hence, $S-\Gamma$ is disconnected and the lemma is proved.

Now we go back to the proof of the proposition.

Proof of the sufficiency. $S$ being a schlicht Riemann surface, then there is

$$
\Phi: S=X_{\mathbf{w}}-\left\{p_{1}, \ldots, p_{N}\right\} \rightarrow \Phi(S)=\overline{\mathbf{C}}-\left\{z_{1}, \ldots, z_{N}\right\}
$$

a conformal representation of $S$ into the Riemann sphere $\overline{\mathbf{C}}$ (see [11]). The function $m(x, y)$ defined in $U-\left(\gamma_{p_{1}} \cup \cdots \cup \gamma_{p_{N}} \cup\{0\}\right)$ by

$$
m(x, y)=\Phi \circ \mathbf{r}(x, y)
$$

and extended to $U-\{0\}$ by $m\left(\gamma_{p_{i}}\right)=z_{i}$ is the desired meromorphic integral.

\section{LINEARIZATION}

We give in this section a topological criterion for a well-behaved differential form $\mathbf{w}$ to be conjugate to a linear form $r y d x-s x d y$, where $r, s$ are coprime positive integers, and then as an application we characterize the germs at $0 \in \mathbf{C}^{2}$ of pure meromorphic functions that are conjugate to the functions $R\left(x^{r} / y^{s}\right)$, where $R$ is a rational function on $\overline{\mathbf{C}}$. Recall that two germs at $0 \in \mathbf{C}^{2}$ of differential forms $\mathbf{w}, \mathbf{w}^{\prime}$ (resp. of meromorphic functions $m, m^{\prime}$ ) are said to be conjugate if there is a germ of biholomorphism $\Phi:\left(\mathbf{C}^{2}, 0\right) \rightarrow\left(\mathbf{C}^{2}, 0\right)$ such that

$$
\left.\Phi^{*} \mathbf{w} \wedge \mathbf{w}^{\prime}=0 \quad \text { (resp. } m \circ \Phi=m^{\prime}\right) .
$$

The notation $X_{w}$ will have the same meaning as in the previous section. The linearization theorem is the following: 
Theorem 4.1. A differential form $\mathbf{w}$ is conjugate to a linear form $r y d x-s x d y$, where $r, s$ are coprime positive integers, if and only if $X_{\mathbf{w}}$ is a Hausdorff space. Proof of necessity. When $\mathbf{w}$ is conjugate to $r y d x-s x d y$, then the orbit space $X_{\mathrm{w}}$ is homeomorphic to $X_{(r y d x-s x d y)}$. But $r y d x-s x d y$ admits $x^{r} / y^{s}$ as a meromorphic integral. Since $r, s$ are coprime, then for every $c \in \mathbf{C}^{*}$, the variety $V_{c}=\left\{x^{r}-c y^{s}=0\right\}$ is irreducible, i.e. $V_{c}-\{0\}$ is connected. We have then $X_{(r y d x-s x d y)}$ homeomorphic to $\overline{\mathbf{C}}$ and the necessity is proved.

The proof of the sufficiency, given at the end of this section, is based upon the following proposition

Proposition 4.1. Assume that $x_{\mathbf{w}}$ is a Hausdorff space. Then $\mathbf{w}$ has order 1 at 0 .

Proof of the proposition in the dicritic case. Assume that

$$
\mathbf{w}(x, y)=\left(a_{\nu}(x, y)+\alpha(x, y)\right) d x+\left(b_{\nu}(x, y)+\beta(x, y)\right) d y,
$$

where $\nu$ is the order at 0 of $\mathbf{w}$ and $a_{\nu}(x, y) d x+b_{\nu}(x, y) d y$ is its $\nu$ th jet, is dicritic, i.e.

$$
x a_{\nu}(x, y)+y b_{\nu}(x, y)=0 .
$$

Then $E_{0}^{*} \mathbf{w}(x, t)=x^{\nu+1} \widetilde{\mathbf{w}}_{1}(x, t)$ and $E_{0}^{*} \mathbf{w}(s, y)=y^{\nu+1} \widetilde{\mathbf{w}}_{2}(s, y)$, where $E_{0}^{*}: \mathbf{C}^{2}$ $\rightarrow \mathbf{C}^{2}$ is the blow-up map and where

$$
\begin{aligned}
& \widetilde{\mathbf{w}}_{1}(x, t)=\frac{\alpha(x, t x)+t \beta(x, t x)}{x^{\nu+1}} d x+\left(b_{\nu}(1, t)+\frac{\beta(x, t x)}{x^{\nu}}\right) d t \\
& \widetilde{\mathbf{w}}_{2}(s, y)=\frac{\beta(s y, y)+s \alpha(s y, y)}{y^{\nu+1}} d y+\left(a_{\nu}(s, 1)+\frac{\alpha(s y, y)}{y^{\nu}}\right) d s .
\end{aligned}
$$

We first show that $b_{\nu}(1, t)$ is constant. If not, assume that $b_{\nu}(1, t)$ has a root $t_{0}$ and consider an integral $m(x, y)$ of $\mathbf{w}$ that separates the integral curves (see Proposition 3.1). Then the function $\widetilde{m}$ defined in a neighborhood of $E_{0}^{-1}(0)$ by

$$
\widetilde{m}(x, t)=m(x, t x) \quad \text { and } \quad \tilde{m}(s, y)=m(s y, y)
$$

is an integral of $E_{0}^{*} \mathbf{w}$ that satisfies $(\partial \tilde{m} / \partial t)\left(0, t_{0}\right)=0$. This means that the rational function $\widetilde{m}(0, t)$ on the sphere $\overline{\mathbf{C}}\left(=E_{0}^{-1}(0)\right)$ is not injective and therefore $\tilde{m}$, and by consequence $m$, does not separate the integral curves. Thus the assumption is false and so $b_{\nu}(1, t)=C$, where $C$ is a constant. Similarly, we show that $a_{\nu}(s, 1)=C^{\prime}$. Hence

$$
a_{\nu}(x, y)=C^{\prime} y^{\nu} \quad \text { and } \quad b_{\nu}(x, y)=C x^{\nu} .
$$

Finally, it follows from (4.1) and (4.2) that $C=-C^{\prime}$ and $\nu=1$.

Some lemmas are needed for the proof of the proposition in the nondicritic case. 
Lemma 4.1. Assume that $X_{\mathrm{w}}$ is a Hausdorff space and $\mathbf{w}$ nondicritic. Then the foliation $\overline{\mathbf{F}}_{E_{0}^{*} \mathbf{w}}$ has two singularities on $E_{0}^{-1}(0)$. Moreover we can assume that these singularities are $N=(x=0, t=0)$ and $S=(s=0, y=0)$.

Proof. The foliation $\overline{\mathbf{F}}_{E_{0}^{*} \mathbf{w}}$ is defined in the charts $(x, t),(s, y)$ by

$$
\begin{aligned}
\widetilde{\mathbf{w}}_{1}(x, t)= & \left(\left(a_{\nu}(1, t)+t b_{\nu}(1, t)\right)+\frac{\alpha(x, t x)+t \beta(x, t x)}{x^{\nu}}\right) d x \\
& +x\left(b_{\nu}(1, t)+\frac{\beta(x, t x)}{x^{\nu}}\right) d t \\
\widetilde{\mathbf{w}}_{2}(s, y)= & \left(\left(b_{\nu}(s, 1)+s a_{\nu}(s, 1)\right)+\frac{\beta(s y, y)+s \alpha(s y, y)}{y^{\nu}}\right) d y \\
& +y\left(a_{\nu}(s, 1)+\frac{(s y, y)}{y^{\nu}}\right) d s .
\end{aligned}
$$

We can assume, by a linear change of variables, that $s=0$ is not a root of $b_{\nu}(s, 1)+s a_{\nu}(s, 1)=0$, so that the singularities of $\mathbf{F}_{E_{0}^{*} \mathbf{w}}$ are the roots $t_{1}, \ldots, t_{n}$ of $a_{\nu}(1, t)+t b_{\nu}(1, t)=0$. Since $\mathbf{w}$ is nondicritic, then $S=$ $\mathbf{C P}^{1}-\left\{t_{1}, \ldots, t_{n}\right\}$ is a leaf of $\overline{\mathbf{F}}_{E_{0}^{*} \mathbf{w}}$. Moreover, the fact that $\mathbf{w}$ is well behaved implies that all the leaves $\Gamma_{p}$ of $\overline{\mathbf{F}}_{E_{0}^{*} \mathbf{w}}$ adhere to $\mathbf{C} \mathbf{P}^{1}\left(=E_{0}^{-1}(0)\right)$. Hence, these leaves accumulate to the singularities $t_{1}, \ldots, t_{n}$ (w nondicritic). So we can assume that in a neighborhood $O_{1}$ of $t_{1} \in \mathrm{C}_{0}^{2}$ all the leaves $\Gamma_{p}, p \in O_{1}$, accumulate at $t_{1}$. Let us show that in this situation $E_{0}^{*} \mathbf{w}$ has a holomorphic integral in the neighborhoods of the remaining singularities $t_{2}, \ldots, t_{n}$. For, assume that $\overline{\mathbf{F}}_{E_{0}^{*} \mathbf{w}}$ has a pure meromorphic integral in a neighborhood $O_{i}$ of a singularity $t_{i}$ with $i \geq 2$. Then the function $\widetilde{m}$ defined by

$$
\widetilde{m}(x, t)=m(x, t x) \quad \text { and } \quad \tilde{m}(s, y)=m(s y, y),
$$

where $m$ is a pure meromorphic integral as in Proposition 3.1, would be a pure meromorphic function at $t_{1}$ and at $t_{i}$. But the fact that the germ of a pure meromorphic function assumes all the values of $\overline{\mathbf{C}}$ would then imply that distinct leaves $\Gamma_{p_{1}}$ and $\Gamma_{p_{i}}$ (in neighborhoods of $t_{1}$ and $t_{i}$ respectively) are not separated by $\widetilde{m}$. Hence, the distinct integral curves $\gamma_{E_{0}\left(p_{1}\right)}$ and $\gamma_{E_{0}\left(p_{i}\right)}$ of w would not be separated by $m$, and this contradicts the definition of $m$.

Now that the holomorphic integrability of the differential form $E_{0}^{*} \mathbf{w}$ is established at $t_{2}, \ldots, t_{n}$, we show that $n \leq 2$. If $n$ were $\geq 3$, then one could find distinct leaves $\Gamma_{p_{2}}, \ldots, \Gamma_{p_{n}}$ such that $\Gamma_{p_{i}} \neq S$ for $i=2, \ldots, n$ and $\Gamma_{p_{i}}$ accumulates to $t_{i}$ (if $S$ were the only leaf that accumulates to $t_{i}$, then $t_{i}$ would not be a singularity). Then $\widetilde{m}\left(\Gamma_{p_{i}}\right)=m(S), i=2, \ldots, n$, and again $m$ would not separate the distinct leaves $\gamma_{E_{0}\left(p_{2}\right)}, \ldots, \Gamma_{E_{0}\left(p_{n}\right)}$.

Finally to prove that $n=2$, assume that $n=1$. Then $S=\mathbf{C P}^{1}-\left\{t_{1}\right\}$ would be a leaf of $\mathbf{F}_{E_{0}^{*} \mathbf{w}}$ and two possibilities would occur at $t_{1}$ : either the orbit space $X_{\widetilde{w}_{1}}$ of $\widetilde{\mathbf{w}}_{1}$ at $t_{1}$ is Hausdorff or not. In the case where it is a Hausdorff space, 
the foliation $\overline{\mathbf{F}}_{E_{0}^{*} \mathbf{w}}$ would have a meromorphic integral $F$ that would separate all its leaves and so if $c_{0}=F(S)$, then the function $m=F \circ E_{0}^{-1}$ would be a pure meromorphic integral of $\mathbf{w}$ that avoids the value $c_{0}$ and this is impossible. If $X_{\widetilde{w}_{1}}$ is not a Hausdorff space, then any meromorphic integral of $\widetilde{\mathbf{w}}_{1}$ would not separate the leaves and so $\mathbf{w}$ would not have a meromorphic integral that would separate its integral curves. Therefore $n=2$.

The second part of the lemma can be proved as follows:

$$
\begin{aligned}
\psi: \overline{\mathbf{C}} \times \mathbf{C} \cong \mathbf{C}_{0}^{2} & \rightarrow \overline{\mathbf{C}} \times \mathbf{C} \cong \mathbf{C}_{0}^{2}, \\
(\zeta, z) & \mapsto(H(\zeta), z),
\end{aligned}
$$

where $H: \overline{\mathbf{C}} \rightarrow \overline{\mathbf{C}}$ is the Mobius transformation $H(\zeta)=\left(\zeta-t_{1}\right) /\left(\zeta-t_{2}\right)$, is a biholomorphism of $\mathbf{C}_{0}^{2}$ and $\psi^{*} \widetilde{\mathbf{w}}_{1}$ has singularities at $N$ and $S$.

Lemma 4.2. Assume that the differential form $\mathrm{w}$ of order $\nu$ at 0 has an integral ( holomorphic or meromorphic). Then its $\nu$-jet $\mathbf{w}_{\nu}$ has an integral.

Proof. If $f$ is a holomorphic function of order $k$ at 0 , then denote by $f_{k}$ its $k$-jet. Then

$$
d f \wedge \mathbf{w}=d f_{k} \wedge \mathbf{w}_{\nu}+\eta,
$$

where $d f_{k} \wedge \mathbf{w}_{\nu}$ is a homogeneous 2-form of order $k+\nu-1$ and $\eta$ a 2-form with order $\geq \nu+k$. In particular, when $f$ is an integral of $\mathbf{w}$ we get $d f_{k} \wedge \mathbf{w}_{\nu}=0$, i.e. $f_{k}$ is an integral of $\mathbf{w}_{\nu}$.

If $m=\alpha / \beta$ is a pure meromorphic function, we write

$$
\alpha=\alpha_{k}+\left(\alpha-\alpha_{k}\right) \text { and } \beta=\beta_{j}+\left(\beta-\beta_{j}\right),
$$

where $k, j$ are the respective orders of $\alpha, \beta$ and $\alpha_{k}, \beta_{j}$ their respective jets of order $k, j$. We can assume, by adding a constant to $m$ if necessary, that $\alpha_{k} / \beta_{j}$ is not constant. Then $\beta_{j} d \alpha_{k}-d \alpha_{k} \beta_{j}$ is homogeneous of order $k+j-1$. Hence,

$$
(\beta d \alpha-\alpha d \beta) \wedge \mathbf{w}=\left(\beta_{j} d \alpha_{k}-\alpha_{k} d \beta_{j}\right) \wedge \mathbf{w}_{\nu}+\eta,
$$

where $\eta$ is a 2 -form with order $\geq \nu+k+j$. In particular, when $m$ is an integral of $\mathbf{w}$, we get $\left(\beta_{j} d \alpha_{k}-\alpha_{k} d \beta_{j}\right) \wedge \mathbf{w}_{\nu}=0$, i.e. $\alpha_{k} / \beta_{j}$ is an integral of $\mathbf{w}_{\nu}$. The lemma is thus proved.

Lemma 4.3. Assume that $\mathbf{w}$ has an integral (holomorphic or meromorphic) and that $\overline{\mathbf{F}}_{E_{0}^{*} \mathbf{w}}$ has only singularities of order 1 at $N=(x=0, t=0)$ and $S=$ $(s=0, y=0)$. Then $\mathbf{w}$ has order 1 at 0 .

Proof. The leaves of $\overline{\mathbf{F}}_{E_{0}^{*} \mathbf{w}}$ are the integral curves of the differential forms $\widetilde{\mathbf{w}}_{1}(x, t)$ and $\widetilde{\mathbf{w}}_{2}(s, y)$ given by (4.3). We can assume, after a linear change of variables, that

$$
\frac{\alpha(x, t x)}{x^{\nu+1}}(x=0, t=0)=0 \quad \text { and } \quad \frac{\beta(s y, y)}{y^{\nu+1}}(s=0, y=0)=0 .
$$


Since the differential form $\widetilde{\mathbf{w}}_{1}(x, t)$ has only one singularity of order 1 at $N$, then

$$
a_{\nu}(1, t)+t b_{\nu}(1, t)=c t^{k},
$$

for some integer $k \leq \nu+1$ and a nonzero constant $c$. The fact that $\widetilde{\mathbf{w}}_{1}$ has an integral and has order 1 at $N$ implies, by Lemma 4.2, that the first jet $J^{1} \widetilde{\mathbf{w}}_{1}$ has an integral at $N$. Furthermore, since a singular foliation defined by a linear form $c_{1} y d x+c_{2} x d y$ has a meromorphic integral if and only if $c_{1} / c_{2}$ is a rational number, then $k=1$ and $b(1,0) \neq 0$, i.e.

$$
J^{1} \widetilde{\mathbf{w}}_{1}(x, t)=c t d x+x b_{\nu}(1,0) d t .
$$

So we can write

$$
b_{\nu}(1, t)=b_{\nu}(1,0)+Q(t),
$$

where $Q(t)$ is a polynomial of degree $\leq \nu-1$ (because of (4.4) and degree $\left.a_{\nu}(1, t) \leq \nu\right)$, and

$$
a_{\nu}(1, t)=a_{0} t-Q(t),
$$

for some constant $a_{0}$ such that $c=a_{0}+b_{\nu}(1,0) \neq 0$. Hence,

$$
\begin{aligned}
& a_{\nu}(x, y)=a_{0} y x^{\nu-1}-y x^{\nu-1} Q(y / x), \\
& b_{\nu}(x, y)=b_{\nu}(1,0) x^{\nu}+x^{\nu} Q(y / x) .
\end{aligned}
$$

Therefore,

$$
\begin{aligned}
\widetilde{\mathbf{w}}_{2}(s, y)= & \left(\left(a_{0}+b_{\nu}(1,0)\right) s^{\nu}+\frac{\beta(s y, y)+s \alpha(s y, y)}{y^{\nu}}\right) d y \\
& +y\left(a_{0} s^{\nu-1}-s^{\nu-1} Q(1 / s)+\frac{(s y, y)}{y^{\nu}}\right) d s
\end{aligned}
$$

has an integral and is of order 1 at $S=(s=0, y=0)$ only if $\nu=1$. The lemma is proved.

Proof of the proposition in the nondicritic case. The foliation $\overline{\mathbf{F}}_{E_{0}^{*} \mathbf{w}}$ has singularities at $N$ and $S$ (see Lemma 4.1); one of its leaves accumulates at $S$ and all the others at $N$. Since the blow-up of this foliation at $S$ gives rise to a new foliation with only two singularities of the same type as the singularity at $S$, we see then by further blow-ups of the singularities that at the final step, we obtain a reduced foliation with only two singularities on each branch of the exceptional divisor $E_{S}^{-1}(S)$, where $E_{S}$ is the desingularization of $\overline{\mathbf{F}}_{E_{0}^{*} \mathbf{w}}$ at $S$. Hence, by blow-downs, we see that $\overline{\mathbf{F}}_{E_{0}^{*} w}$ has order 1 at $S$ (Lemma 4.3). Next, since the orbit space $X_{\widetilde{\mathbf{w}}_{1}}$ is Hausdorff (because $X_{\mathbf{w}}$ is ), then either $\widetilde{\mathbf{w}}_{1}$ is dicritic at $N$, in which case it has order 1 , or else its blow-up gives rise to a new foliation of the same type as $\overline{\mathbf{F}}_{E_{0}^{*} \mathbf{w}}$. Since this process of blow-ups eventually reaches the dicritic situation (Theorem 2.12), then we conclude, after a succession of blow-downs, that $\widetilde{\mathbf{w}}_{1}$ has order 1 at $N$. Hence, Lemma 4.3 insures that $\mathbf{w}$ has order 1 at 0 and the proposition is proved. 
Proof of Theorem. 4.1 It follows from Proposition 4.1 that $\mathbf{w}$ has order 1 at 0 and from Proposition 3.1 that $\mathbf{w}$ has a pure meromorphic integral. Then we can write

$$
\mathbf{w}(x, y)=(r y d x-s x d y)+\eta,
$$

where $r, s$ are coprime positive integers and $\eta$ a differential form with order $\geq 2$ at 0 . Now a classical theorem due to H. Poincare [8] says that when $\mathbf{w}$ has the form (4.5), it is conjugate to its first jet $(r y d x-s x d y)$. The theorem is thus proved.

As a consequence of Theorems 4.1 and 1.43, we obtain a rationalization result for a class of germs of meromorphic functions. Consider a germ at $0 \in \mathbf{C}^{2}$ of a pure meromorphic function $m(x, y)$ and denote by $X_{m}$ the orbit space of the foliation defined by the level sets of $m$. Then

Theorem 4.2. A germ at $0 \in \mathbf{C}^{2}$ of a pure meromorphic function $m$ is conjugate to a rational function of the form $R\left(x^{r} / y^{s}\right)$, where $R$ is a rational function on the sphere $\overline{\mathbf{C}}$ and $r, s$ are coprime positive integers, if and only if $X_{m}$ is a Hausdorff space.

\section{NONEXISTENCE OF A TOPOLOGICAL CRITERION FOR TRANSCENDENTAL INTEGRABILITY}

In [12] and [13] M. Suzuki shows that the differential form

$$
\mathbf{u}(x, y)=\left(y^{3}+y^{2}-x y\right) d x-\left(2 x y^{2}+x y-x^{2}\right) d y
$$

is well-behaved and does not have a meromorphic integral. However the function $(x / y) \exp \left(\left(y^{2}+y\right) / x\right)$ is a transcendental integral of $\mathbf{u}$.

Here, we given an example of a well-behaved differential form $\mathbf{W}$ that does not have a transcendental integral. Then an argument similar to that used by D. Cerveau and J. F. Mattei in [2] to prove that $\mathbf{u}$ is topologically conjugate to the differential form

$$
\mathbf{v}(x, y)=\left(2 y^{2}+x^{3}\right) d x-2 x y d y,
$$

for which $\left(y^{2}-x^{3}\right) / x^{2}$ is a meromorphic integral, shows that $\mathbf{u}, \mathbf{v}$ and $\mathbf{W}$ are topologically conjugate. Two germs at $0 \in \mathbf{C}^{2}$ of holomorphic differential forms are said to be topologically conjugate, if there is a homeomorphism of a neighborhood of 0 that maps the integral curves of the first differential form into that of the second. Hence we conclude: there is no topological criterion for the existence of transcendental integrals of a germ of a holomorphic differential form in $\mathbf{C}^{2}$.

Let

$$
\mathbf{W}(x, y)=\left(x y-c y^{2}-c y^{3}\right) d x+\left(-x^{2}+c x y+2 c x y^{2}-y^{3}-y^{4}\right) d y,
$$

where $c=(\sqrt{2}+1) /(\sqrt{2}-1)$. In the rest of this section we show the following: 
Proposition 5.1. $\mathrm{W}$ is well behaved and does not have a transcendental integral.

The proof of this proposition is based upon some lemmas.

Lemma 5.1. $\mathbf{W}$ is well behaved.

Proof. We use the blow-up map $E_{0}$. We see that

$$
E_{0}^{*} \mathbf{W}(x, t)=x^{3} \widetilde{\mathbf{W}}_{1}(x, t) \quad \text { and } \quad E_{0}^{*} \mathbf{W}(s, y)=y^{3} \widetilde{\mathbf{W}}_{2}(s, y),
$$

where

$$
\widetilde{\mathbf{W}}_{1}(x, t)=\left(c t^{3}-t^{4}-t^{5} x\right) d x+\left(-1+c t+2 c t^{2} x-t^{3} x-t^{4} x^{2}\right) d t
$$

and

$$
\widetilde{\mathbf{W}}_{2}(s, y)=(c s-(1+y)) d y+(s-c(1+y)) d s .
$$

The differential form $\widetilde{\mathbf{W}}_{1}$ (resp. $\widetilde{\mathbf{W}}_{2}$ ) does not vanish on the complex curve $\{x=0\}$ (resp. $\{y=0\}$ ). So the foliation $\overline{\mathbf{F}}_{E_{0}^{*} \mathbf{w}}$ has no singularities on $E_{0}^{-1}(0)$. Moreover, $E_{0}^{-1}(0)$ is transversal to all the leaves of $\overline{\mathbf{F}}_{E_{0}^{*} \mathbf{w}}$ except one: it is tangent to the leaf that passes through the point $(s=c, y=0)$. Next, since $E_{0}^{-1}$ maps the leaves of $\mathbf{F}_{\mathbf{w}}$ into that of $\overline{\mathbf{F}}_{E_{0}^{*} \mathbf{W}}$, then the integral curves of $\mathbf{W}$ adhere to 0 and they are closed in $U-\{0\}$, provided that $U$ is a small neighborhood of 0 . That is, $\mathbf{W}$ is well behaved and the lemma is proved.

Lemma 5.2. W does not have a meromorphic integral.

Proof. We use an argument similar to that used by M. Suzuki in [12]. The linear form $\widetilde{\mathbf{W}}_{2}(s, y)$ has a singularity at $(s=0, y=-1)$ and in the coordinates $(Y=s+(y+1), S=s-(1+y)), \widetilde{\mathbf{W}}_{2}$ has the expression

$$
\widetilde{\mathbf{W}}_{2}(S, Y)=\frac{1}{\sqrt{2}-1}(\sqrt{2} S d Y-Y d S) .
$$

By integrating the corresponding linear differential equation, we see that the integral curves of $\widetilde{\mathbf{W}}_{2}$ are not closed near $(s=0, y=-1)$. Thus $\widetilde{\mathbf{W}}_{2}$ has no meromorphic integral near its singularity. Now, assume that $\mathbf{W}$ has a meromorphic integral $m(x, y)$ in a neighborhood of $0 \in \mathbf{C}^{2}$. Then the function $\tilde{m}$ defined in a neighborhood of $E_{0}^{-1}(0) \subset \mathbf{C}_{0}^{2}$ by

$$
\widetilde{m}(x, t)=m(x, t x) \quad \text { and } \quad \tilde{m}(s, y)=m(s y, y)
$$

would be an integral of $E_{0}^{*} \mathbf{W}$. The integral curve of $\widetilde{\mathbf{W}}_{2}$ through a point $A$ with coordinates $(s=a, y=-1), a \neq 0$, intersects the $s$-axis at a point $B$. Let $L$ be a (real) path that joins $A$ to $B$ in the integral curve through $A$ of $\widetilde{\mathbf{W}}_{2}$. Then we could choose $\varepsilon$ small and $R$ large so that the bidisc

$$
D(0, R) \times D(0, \varepsilon) \subset \mathbf{C}_{s} \times \mathbf{C}_{y}
$$

is contained in the domain of $\tilde{m}$ and furthermore, the projection of the path $L$ into the $s$-axis is contained in the disc $D(0, R)$. The function $\widetilde{m}$ would extend, by constancy along the integral curves, to a neighborhood $V$ of $L$. We 
can then assume, by shrinking $V$ about $L$ if necessary, that the projection of $V$ into the $s$-axis is also contained in the disc $D(0, R)$. Hence, $\widetilde{m}$ would be a holomorphic function (into $\overline{\mathbf{C}}$ ) in

$$
(D(0, R) \times D(0, \varepsilon)) \cup V,
$$

whose envelope of holomorphy is

$$
D(0, R) \times\left(D(0, \varepsilon) \cup V^{\prime}\right),
$$

where $V^{\prime}$ is the projection of $V$ into the $y$-axis. Therefore, $\tilde{m}$ would extend as a meromorphic integral of $\widetilde{\mathbf{W}}_{2}(s, y)$ in a neighborhood of the singularity $(s=0, y=-1)$. This is impossible and the lemma is thus proved.

In what follows multiform functions are going to be considered. Let us recall this notion. Let $U$ be a complex manifold and let $\mathbf{q}: \mathbf{U} \rightarrow U$ be the universal covering of $U$. A multiform (or a multivalued) function on $U$ is a relation $m: U \rightarrow \overline{\mathbf{C}}$ such that $m \circ q$ is a holomorphic function (into $\overline{\mathbf{C}}$ ) defined in $\mathbf{U}$.

Now, consider the multiform function $m(\zeta)=(\zeta+1)^{\sqrt{2}} /(\zeta-1)$ defined in $\mathbf{C P} \mathbf{P}^{1}-\{-1,1, \infty\}$. Let $\mathbf{q}: D \rightarrow \mathbf{C P}^{1}-\{-1,1, \infty\} \quad(D$ denotes the unit disc) be the universal covering and $\widetilde{m}$ be the lift to $D$ of $m$. An invariance function of $\tilde{m}$ is an (global) analytic function $\rho$ that satisfies

$$
\tilde{m}(\rho(z))=m(z) \quad \text { for every } z \in D .
$$

Denote by $S$ the Riemann surface of $\rho$ and by $\mathbf{p}$ the projection into $D$.

Lemma 5.3. There is a sequence of points $\left(\alpha_{k, j}\right)_{k, j \in \mathbf{Z}^{+}}$in $D$ such that: $S$ is a covering of $D-\left\{\alpha_{k, j} ; k, j \in \mathbf{Z}^{+}\right\}$and for every $\beta \in \mathbf{p}^{-1}\left(\alpha_{k, j}\right)$ there are $U_{k, j}$ ( resp. $V_{k, j}$ ) neighborhood of $\beta \in S$ (resp. $\alpha_{k, j} \in D$ ), and $\Phi_{k, j}$ (resp. $\psi_{k, j}$ ) homeomorphism of $U_{k, j}$ (resp. $V_{k, j}$ ) into $\mathbf{C}$, such that

$$
\psi_{k, j} \circ \mathbf{p} \circ \Phi_{k, j}^{-1}(z)=z^{\nu}
$$

where $\nu=1$ or 2 .

Proof. For any branch of $m$, we have

$$
m^{\prime}(\zeta) \neq 0 \quad \text { if } \zeta \neq c, \quad m^{\prime}(c)=0 \quad \text { and } \quad m^{\prime \prime}(c) \neq 0
$$

where $c=(\sqrt{2}+1) /(\sqrt{2}-1)$. Denote by $\left(\alpha_{k}\right)_{k \in \mathbf{Z}^{+}}$the infinite sequence $\mathbf{p}^{-1}(c)$. Then it follows from $(5.1)$ that

$$
\tilde{m}^{\prime}(z) \neq 0 \quad \text { if } z \neq \alpha_{k}, \quad \tilde{m}^{\prime}\left(\alpha_{k}\right)=0 \quad \text { and } \quad \tilde{m}^{\prime \prime}\left(\alpha_{k}\right) \neq 0
$$

So that $\tilde{m}$ is a covering from $\mathbf{G}=D-m^{-1}\left\{m\left(\alpha_{k}\right), k \in \mathbf{Z}^{+}\right\}$into its image. An invariance function $\rho$ of $\widetilde{m}$ can then be continued throughout $\mathbf{G}$. Next, for every $k \in \mathbf{Z}^{+}$, denote by $\left(\alpha_{k, j}\right)_{j \in \mathbf{Z}^{+}}$the infinite sequence $\widetilde{m}^{-1}\left(\widetilde{m}\left(\alpha_{k}\right)\right)-\left\{\alpha_{k}\right\}$. Now, let $k$ and $j$ be fixed. Then when $z$ tends to $\alpha_{k, j}$, a branch of $\rho$ tends either to $\alpha_{k, j^{\prime}}$ (for some $j^{\prime}$ ) or to $\alpha_{k}$. Since $m$ is a local biholomorphism at $\alpha_{k, j}$ and $\alpha_{k, j^{\prime}}$, then the branch of $\rho$ that tends to $\alpha_{k, j^{\prime}}$ can be extended 
through $\alpha_{k, j}$. That is, there are neighborhoods $U_{k, j}$ of the corresponding point to $\alpha_{k, j}$ in $S$, and $V_{k, j}$ of $\alpha_{k, j}$ in $D$, and homeomorphisms $\Phi_{k, j}$ and $\psi_{k, j}$ of $U_{k, j}$ and $V_{k, j}$ respectively, into $\mathbf{C}$, such that

$$
\psi_{k, j} \circ \mathbf{p} \circ \Phi_{k, j}^{-1}(z)=z .
$$

In the case where $\rho(z)$ tends to $\alpha_{k}$, then it follows from

$$
\widetilde{m}\left(\alpha_{k}\right)=\tilde{m}\left(\alpha_{k, j}\right), \quad \tilde{m}^{\prime}\left(\alpha_{k, j}\right) \neq 0, \quad \tilde{m}^{\prime}\left(\alpha_{k}\right)=0 \quad \text { and } \quad \tilde{m}^{\prime \prime}\left(\alpha_{k}\right) \neq 0,
$$

the existence of neighborhoods $V_{k}$ and $V_{k, j}$ of $\alpha_{k}$ and $\alpha_{k, j}$ respectively, such that the function

$$
\tilde{m}^{-1} \circ \widetilde{m}: V_{k} \rightarrow \widetilde{m}\left(V_{k}\right) \rightarrow V_{k, j}
$$

is conjugate to $z^{2}$. That is, $\rho(z)$ is conjugate to $\sqrt{z}$ near $\alpha_{k, j}$ and the lemma is proved.

Let $\rho$ be an invariance function of $\widetilde{m}$ distinct from the identity. Then define the function $H$, multiform on $\mathbf{C} \mathbf{P}^{1}-\{-1,1, \infty\}$, by $H=\mathbf{q} \circ \rho \circ \mathbf{q}^{-1}$. $H$ is an invariance function of the multiform function $m$. That is, $m \circ H=m$ globally.

Remark 5.1. The integral curve $\Gamma_{(0, c)}$ of the linear form $\widetilde{\mathbf{W}}_{2}(s, y)$ through $(s=c, y=0)$ is tangent to $\mathbf{C P}^{1}$. So, when $\zeta$ is close to $c$ the integral curve $\Gamma_{(0, \zeta)}$ intersects $\mathbf{C P}^{1}$ at $\zeta$ and another point $\zeta^{\prime}$. The mapping $J: \zeta \longmapsto \zeta^{\prime}$ is a well-defined holomorphic function near $c$. Moreover, the multiform function $H$ is the analytic continuation of $J$. To see why, notice that $m(\zeta)$ is the restriction to $\mathbf{C} \mathbf{P}^{1}$ of

$$
\mu(s, y)=\frac{(s+(1+y)) \sqrt{2}}{(s-(1+y))},
$$

a multiform integral of $\widetilde{\mathbf{w}}_{2}(s, y)$ and $J$ is the invariance function of any branch of $m$.

Remark 5.2. It follows from $J \circ J(\zeta)=\zeta$ that

$$
H \circ H=\text { id, }
$$

$H$ considered as a global analytic function.

Lemma 5.4. $H$ is not an algebraic function.

Proof. If $H$ were an algebraic function, then it would follow from (5.2) that the space $\mathbf{R}$, obtained by identifying the points in $\mathbf{C P}^{1}$ via the equivalence relation

$$
\zeta \sim \zeta^{\prime} \quad \text { iff } \quad \zeta^{\prime} \in H(\zeta)
$$

is a compact Riemann surface. So, if $\mathbf{r}: \mathbf{C P}^{1} \rightarrow \mathbf{R}$ is the quotient map, $\Phi$ any nonconstant meromorphic function on $\mathbf{R}$ (such function always exists on a compact Riemann surface [11]) and $\psi: \widetilde{U} \rightarrow \mathbf{C P}^{1}$, where $\widetilde{U}$ is a tubular 
neighborhood of $\mathbf{C} \mathbf{P}^{1} \subset \mathbf{C}_{0}^{2}$, is defined by $\psi(p)=\Gamma_{p} \cap \mathbf{C} \mathbf{P}^{1}$, with $\Gamma_{p}$ the leaf through $p$ of $\overline{\mathbf{F}}_{E_{0}^{*} \mathbf{w}}$, then the mapping

$$
\mu(x, y)=\Phi \circ \mathbf{r} \circ \psi \circ E_{0}^{-1}(x, y)
$$

would be a meromorphic integral of $\mathbf{W}$. This contradicts Lemma 5.2 and Lemma 5.4 is proved.

Define the function $\vartheta: \mathbf{C P}^{1} \rightarrow \overline{\mathbb{R}}$ by

$$
\vartheta(s)=\frac{|s+1| \sqrt{2}}{|s-1|} \quad \text { and } \quad \vartheta(t)=|1+t|^{\sqrt{2}}|t|^{1-\sqrt{2}}|1-t|^{-1},
$$

where $t, s$ are the homogeneous coordinates of $\mathbf{C P}$. The function $\vartheta$ is the absolute value of $m$ and its level sets are closed curves in $\mathbf{C P}^{1}$.

Corollary 5.1. For every $\zeta \in \mathbf{C P}^{1}-\{-1,1, \infty\}$, the set $H(\zeta)$ has an accumulation point $\zeta_{\infty}$ on the curve $\vartheta^{-1}(\vartheta(\zeta))$.

Proof. Since $\vartheta$ is the absolute value of $m$ and $H$ is an invariance function of $m$, then $H(\zeta) \subset \vartheta^{-1}(\vartheta(\zeta))$. Since $H$ is not an algebraic function, then $H(\zeta)$ consists of an infinite number of points and the conclusion follows.

Proof of the proposition. If $T(x, y)$ were a transcendental integral of $\mathbf{W}$ in a neighborhood $U$ of $0 \in \mathbf{C}^{2}$ (see Definition 1.41) then the function $\widetilde{T}$, defined in $E_{0}^{-1}(U)$ by

$$
\tilde{T}(x, t)=T(x, t x) \text { and } \quad \tilde{T}(s, y)=T(s y, y),
$$

would be an integral of $E_{0}^{*} \mathbf{W}$, outside finitely many leaves. The restriction $\widetilde{T}_{0}$ of $\widetilde{T}$ to $\mathbf{C} \mathbf{P}^{1}$ would then be a holomorphic function with a finite number of essential singularities. Now, since $\zeta$ and $J(\zeta)$ are on the same leaf (see Remark 5.1), then $\widetilde{T}_{0} \circ J=\widetilde{T}_{0}$ and, by analytic continuation, we get

$$
\widetilde{T}_{0}(H(\zeta))=T_{0}(\zeta) \text { for every } \zeta \in \mathbf{C P}^{1}-\{-1,1, \infty\}
$$

Hence, $\widetilde{T}_{0}$ would have an essential singularity at $\zeta_{\infty}$ (see Corollary 5.1 ). But the number of such accumulation points $\zeta_{\infty}$ is infinite, thus the assumption is contradicted and the proposition is proved.

\section{REFERENCES}

1. C. Camacho and P. Sad, Pontos singulares de equaçoes diferenciais analiticas, $16^{\circ}$ Colóquio Brasileiro de Matemáticas, IMPA.

2. D. Cerveau and J. F. Mattei, Formes integrables holomorphes singulieres, Asterisque, no. 97, Soc. Math. France, Paris, 1982.

3. R. C. Gunning and H. Rossi, Analytic functions of several complex variables, Prentice-Hall, Englewood Cliffs, N.J., 1965.

4. L. Kaup and B. Kaup, Holomorphic functions of several complex variables, De Gruyter Stud. in Math., vol. 3, De Gruyter, 1983. 
5. B. Malgrange, Frobenius avec singularitès1: codimension un, Inst. Hautes Études Sci. Publ. Math. 48 (1976), 163-173.

6. J. F. Mattei and R. Moussu, Holonomie et intègrales premieres, Ann. Sci. Ècole Norm. Sup. (4) 13 (1980), 469-523.

7. R. Narasimhan, Introduction to the theory of analytic spaces, Lecture Notes. in Math., vol. 25, Springer-Verlag, 1966.

8. H. Poincaré, Sur les propriétés des functions définies par les equations aux différences partielles, Thèse, Paris, 1879.

9. K. Saito, On a generalization of de Rham lemma, Ann. Inst. Fourier (Grenoble) 26 (1976), 165-170.

10. A. Seidenberg, Reduction of singularities of the differential equation $A d Y=B d X$, Amer. J. Math. (1968), 248-269.

11. G. Springer, Introduction to Riemann surfaces, Addison-Wesley, Reading, Mass., 1957.

12. M. Suzuki, Sur les relations d'equivalence ouvertes dans les espaces analytiques, Ann. Sci. École Norm. Sup. (4) 4 (1974), 531-542.

13. __ Sur les integrales premieres de certain feuilletages analytiques complexes, Lecture Notes in Math., vol. 670, Springer-Verlag, pp. 53-58.

14. H. Whitney, Complex analytic varieties, Addison-Wesley, Reading, Mass., 1972.

Department of Mathematics, Florida International University, Miami, Florida 33199 